\title{
Evaluation of four methods to identify the homozygotic sex chromosome in small populations
}

Charles Christian Riis Hansen ${ }^{1 *}{ }^{*}$, Kristen M. Westfall $^{1,2}$ and Snæbjörn Pálsson ${ }^{1 \dagger}$

\begin{abstract}
Background: Whole genomes are commonly assembled into a collection of scaffolds and often lack annotations of autosomes, sex chromosomes, and organelle genomes (i.e., mitochondrial and chloroplast). As these chromosome types differ in effective population size and can have highly disparate evolutionary histories, it is imperative to take this information into account when analysing genomic variation. Here we assessed the accuracy of four methods for identifying the homogametic sex chromosome in a small population using two whole genome sequences (WGS) and 133 RAD sequences of white-tailed eagles (Haliaeetus albicilla): i) difference in read depth per scaffold in a male and a female, ii) heterozygosity per scaffold in a male and a female, iii) mapping to the reference genome of a related species (chicken) with annotated sex chromosomes, and iv) analysis of SNP-loadings from a principal components analysis (PCA), based on the low-depth RADseq data.

Results: The best performing approach was the reference mapping (method iii), which identified $98.12 \%$ of the expected homogametic sex chromosome (Z). Read depth per scaffold (method i) identified $86.41 \%$ of the homogametic sex chromosome with few false positives. SNP-loading scores (method iv) identified $78.6 \%$ of the Z-chromosome and had a false positive discovery rate of more than $10 \%$. Heterozygosity per scaffold (method ii) did not provide clear results due to a lack of diversity in both the $Z$ and autosomal chromosomes, and potential interference from the heterogametic sex chromosome (W). The evaluation of these methods also revealed $10 \mathrm{Mb}$ of putative PAR and gametologous regions.
\end{abstract}

Conclusion: Identification of the homogametic sex chromosome in a small population is best accomplished by reference mapping or examining differences in read depth between sexes.

Keywords: Homogametic sex chromosome, Population genetics, Non-model organisms, White-tailed eagle

\section{Background}

Inferences about genetic variation, effective population size and population structure from genomic data in species that have heteromorphic sex chromosomes are dependent on their correct identification and other

\footnotetext{
*Correspondence: ccr3@hi.is

${ }^{\dagger}$ Charles Christian Riis Hansen and Snæbjörn Pálsson contributed equally.

${ }^{1}$ Department of Life and Environmental Sciences, University of Iceland,

Reykjavik, Iceland

Full list of author information is available at the end of the article
}

markers from different genomic regions, i.e., autosomes and the plastid genomes. As these different genomic regions typically have different ploidy numbers, substitution rates, and recombination rates, it follows that they will also be variably affected by genetic drift and selection [1]. Such information can be imperative for successful conservation management based on genetic variation and evolutionary studies. Annotating genomic regions can be accomplished either from a high-quality reference genome of the same or a closely related species. Here, we use genomic data from the white-tailed eagle mapped 
to a golden eagle reference genome to determine which scaffolds belong to the $\mathrm{Z}$ and autosomal chromosomes.

Using a different reference genome from the study species is frequently done [2-5] when chromosomal information is lacking. However, there can be drawbacks as related species can differ e.g., in genome size, synteny and other chromosomal rearrangements, or even lack sex chromosomes altogether [6]. Mapping to a closely related genome could also lead to mis-identification of a sequence that is sex-linked in the reference but not in the focal species or missing the sex chromosome content present in the focal that is not in the reference. However, birds are characterized by evolutionary stable chromosomes with rather little variation in genome size compared to other groups [7, 8].

Like many non-model species, the white-tailed eagle (Haliaeetus albicilla) lacks a well annotated genome. The specimens studied here come from a small and geographically isolated population of white-tailed eagles in Iceland, which currently consists of 80 breeding pairs. The population is recovering slowly from a severe bottleneck in population size during the 19th-20th centuries, when the number of breeding pairs were about 20 for more than 50years [9] and is thus expected to have little genetic variation. The golden eagle (Aquila chrysaetos) and the white-tailed eagle are large raptors with a wide distribution in the northern hemisphere $[10,11]$. Currently there are four genome assemblies available for the golden eagle, consisting of 142 (size: $1233.7 \mathrm{Mb}$, N50: $46.9 \mathrm{Mb}$ ); 1142 (size: $1192.7 \mathrm{Mb}, \mathrm{N} 50$ : 9.2 Mb); 35,366 (size: $1196.0 \mathrm{Mb}$, N50: 0.11 Mb); and 42,881 (size: 1548.4Mb, N50: 1.7 Mb) scaffolds, where only the first has scaffolds assigned to chromosomes [12]. Only three fragmented genomes exist for the white-tailed eagle (consisting of 50,905 scaffolds with the size: $1133.5 \mathrm{Mb}$, and N50: $0.05 \mathrm{Mb}$; 35,313 scaffolds with the size: $1196.5 \mathrm{Mb}$ and $\mathrm{N} 50: 0.12 \mathrm{Mb}$; and 6418 scaffolds with the size: $1222.6 \mathrm{Mb}$ and N50: $4.5 \mathrm{Mb}$ ), with no annotated chromosomes [8]. The mitochondrial genomes of both the white-tailed and golden eagle have been identified $[12,13]$. The Z-chromosome has been identified in golden eagle $(88.2 \mathrm{Mb})$ and it is large in comparison with $\mathrm{Z}$ chromosomes in other birds which have been identified (ranging from 37.9 to $195.3 \mathrm{Mb}$ [14-16]) but similar in size to chicken (Gallus gallus, $82.5 \mathrm{Mb}$ [17]). Resolving the chromosomal composition of the white-tailed eagle genome will facilitate research on the genetics and evolutionary history of the species and for other eagle species. Furthermore, assessing the accuracy of methods for identifying the homozygotic sex chromosome may facilitate annotation of genome assemblies in other species characterized by small population sizes. Here we evaluate the success of four methods to identify the Z-chromosome in the small population of white-tailed eagles in Iceland. Sequencing depth (1) and patterns of heterozygosity (2) were analysed in highdepth whole genome sequence data obtained from one male and one female. The golden eagle scaffold reference genome was mapped to a chicken genome (3), and genotypes from low-depth RAD-sequencing data from 133 white-tailed eagles with a principal component analysis (PCA) (4). Our hypothesis is that the use of heterozygosity will be least successful as it will be reduced in the small population.

A recent review describes various methods for identifying sex chromosomes [18]. When template DNA molecules from a genome are sequenced randomly, it is expected that equivalent chromosomal classes will have similar average sequencing depths, and thus the depth can be used to identify different parts of the genome. For example, mitochondrial DNA is expected to have relatively high read depth, due to greater per-cell copy number than the nuclear chromosomes (this also applies to repeated regions). In addition, the sex chromosome found in the homogametic sex (ZZ or XX) is expected to have double the sequencing depth obtained from the heterogametic sex (ZW or XY), in species with differentiated sex chromosomes, as in birds and mammals [19, 20], but not in species with little differentiation between sex chromosomes such as in several fish species [21, 22]. Thus, for example, identification of the Z (and X) chromosome through depth filtering has been successfully applied to flycatchers [23] and humans [24], and depth is also partly used in programmes for discovering the sex chromosomes [25-27].

Sex differences in heterozygosity can also be used to assess which scaffolds belong to the homogametic sex chromosome e.g., [28]. For any given set of individuals from the same population, the Z-chromosome is expected to have fewer heterozygous positions in females $(\mathrm{ZW})$ than in males (ZZ), whereas autosomal scaffolds are expected to have a similar number of heterozygous positions in both sexes.

Several factors can limit the discriminatory power of depth and heterozygosity to identify $Z$ scaffolds when comparing males and females. First, the difference between the sexes will be reduced for scaffolds containing pseudoautosomal (PAR) and gametologous regions (conserved but non-recombining homologous regions). A study on PAR-regions in birds have shown large variation in the size and divergence of W- and Z-chromosomes across species [29], furthermore $\mathrm{Xu}$ and Zhou [19] showed that the W-chromosome has retained its gene function in birds better than the Y-chromosome in mammals and that the proportion of gametologs can be high. Moreover, long runs of homozygosity affecting $\mathrm{Z}$ scaffolds in males and autosomal scaffolds in both sexes, 
due to inbreeding or small population size, can mask the expected pattern of sex differences in heterozygosity. This is expected to be a marked feature of the white-tailed eagles analysed in this study and have a negative impact on how useful the heterozygosity is in identifying the Z-chromosome.

Another approach is to map scaffolds from an incompletely assembled reference genome to a more fully annotated genome from a "closely" related species. Such mapping can be done with several available programs e.g., LASTZ [30], LAST [31] and YASS [32]. The accuracy of chromosomal locations of scaffolds obtained from this approach depends on the evolutionary distance between the two reference genomes, which can differ due to chromosomal translocations, transposed regions, and repetitive regions [33, 34], sometimes even in closely related species [35]. Thus, this method may be only applicable for taxa with relatively stable genomes such as mammals and birds, though some groups of birds have also recently been shown to have dynamic sex chromosomes [36].

In a PCA of genotypes from all scaffolds i.e., belonging to both autosomes and sex-chromosomes, it is possible that one or more principal components (PCs) split males and females, due to sex specific markers on the sex chromosomes, i.e., on $\mathrm{W}$ or to markers on $\mathrm{Z}$ given a double weight in females. It therefore follows that a PCA could be used to identify scaffolds belonging to sex chromosomes, or alternatively to any sex specific markers, much in the same way as for population or group differentiation. Methods based on sex specific markers have been developed $[37,38]$ to identify the $\mathrm{W}$ and have been commonly used in PCR to diagnose sexes [39]. We tested this by examining the loadings of SNPs from a PCA based on low-depth RAD-sequencing data from 133 white-tailed eagles (Fig. S1) - to assess if they contribute to separation along a specific principal axis [40] by sex.

We show that sex differences in sequencing depth and mapping to a more complete reference genome from a related species provide the most effective means to identify $\mathrm{Z}$ chromosome scaffolds in the white-tailed eagles. However, the approaches based on the PCA, and heterozygosity provide valuable additional information and shed light on some key challenges faced by researchers working with genomic data from species with partially assembled reference genomes.

\section{Results}

To assess the accuracy of the four approaches used to identify the Z-chromosomal scaffolds (depth, Heterozygosity, mapping, and PCA), the reference "scaffoldassembled" golden eagle genome was mapped to a newly released "chromosome-assembled" golden eagle genome, to know the position in the genome of the scaffolds. This was used as a baseline ("truth") when evaluating the methods (Fig. 5, Table 2, and Method section).

\section{Depth}

The overall modes of depth, $195 \mathrm{x}$ for the female and $181 \mathrm{x}$ for the male, were used to estimate the relative sequence depth for each position on each scaffold. A clear bimodal distribution of the depths was observed after discarding the shortest scaffolds $(<198,789$ bases, $\log 10<5.29)$ (Fig. 1, Fig. S2, Fig. S3) and a good distinction of the expected values for the Z-chromosome (0.5) and the autosomes (1) for the female was observed (Fig. 1A and S2). As also expected, this was not observed for the male, but a few $\mathrm{Z}$ scaffolds had a ratio of 2 suggesting occurrence of paralogous regions (Fig. 1C). After the removal of the short scaffolds, 257 scaffolds out of the 1141 scaffolds remained, but covering $98.9 \%$ of the full genome in the chromosome-assembled golden eagle genome, which was used as baseline. In the female, 36 scaffolds comprising $\sim 75.2 \mathrm{Mb}$ had a relative depth close to 0.5 (from 0.466 to 0.533 ), all from the golden eagle Z-chromosome. In comparison, 211 scaffolds $(1.0947 \mathrm{~Gb})$ had a relative depth around 1 (from 0.764 to 1.062 ), whereof 207 were autosomal. The remaining four scaffolds (NW_011950951.1, NW_011950990.1, NW_011951047.1 and NW_011951051.1) mapped to the Z chromosome, comprising $\sim 10 \mathrm{Mb}$ or $0.91 \%$ of the scaffolds identified as autosomes (see Table 2 and Table S1 for all numbers).

The expected male to female ratio $\left(r_{m f}\right)$ of sequence depth is 1 for autosomal and 2 for $\mathrm{Z}$ scaffolds. Implementation of $r_{m f}$ for the scaffolds revealed an even clearer split between the $\mathrm{Z}$ and the autosomes (Fig. 1B), particularly after removing the primarily small scaffolds with relative depth outside the credible range of $0.25-1.5$ in either the male or female. This left 618 scaffolds that accounted for $99.53 \%$ of the total sequence (Fig. 1D). Thereof 93 had $r_{m f}>1.5$, consistent with the expected depth of $Z$ scaffolds. Of these, 79 (76.2 Mb) identified as $\mathrm{Z}$ and $14(0.09 \mathrm{Mb})$ as autosomal chromosomes in the golden eagle genome. We observed 525 scaffolds with $r_{m f}<=1.5$, consistent with the expected depth of autosomes. Of these, 512 scaffolds $(1100.7 \mathrm{Mb})$ identified as autosomes and $13(10.05 \mathrm{Mb})$ as $\mathrm{Z}$ in the golden eagle genome (four of these 13 were also scaffolds NW_011950951.1, NW_011950990.1, NW_011951047.1 and NW_011951051.1).

\section{Heterozygosity}

After filtering, where low quality and spurious sites based on deviation from statistical expectation were removed, only $32 \%$ of scaffolds ( 365 of 1141 ), covering $97.5 \%$ of the genome, had at least one heterozygous genotype in either of the two individuals. Slightly fewer heterozygous sites 


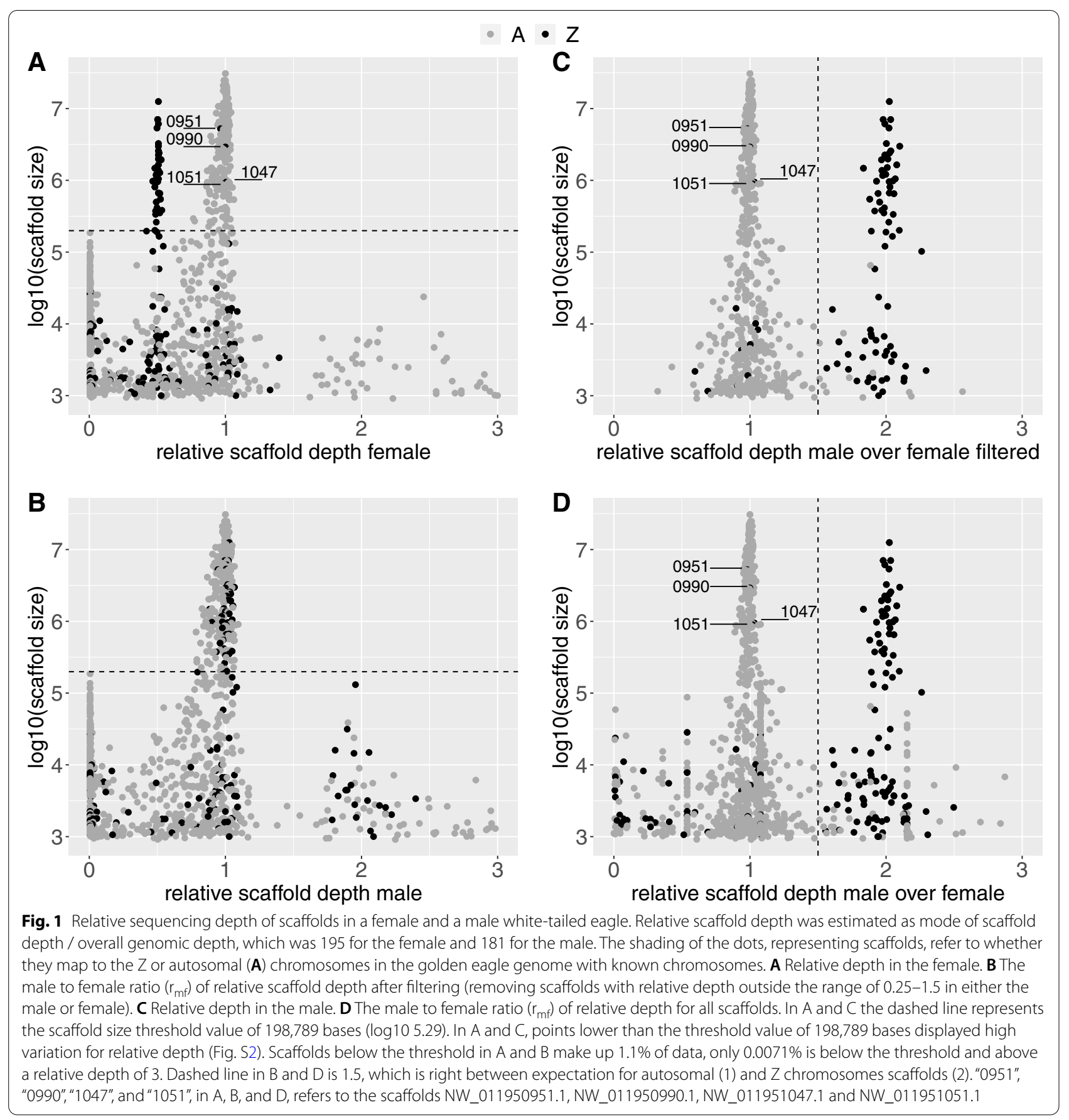

were observed in the female (288) than in the male (300). The majority of the scaffolds with no heterozygous sites mapped to the $\mathrm{Z}$ (80\% in the female, corresponding to $30 \%$ of the $Z$ chromosome; $77 \%$ in the male, covering $23 \%$ of $Z$ ). The $Z$ had generally fewer heterozygous sites after filtering (Table 1, Supplement Figs. 3 and 4), but a majority of the autosomal scaffolds lack heterozygous sites (67, $1.1 \%$ in size). Furthermore, there were more autosomal scaffolds than Z's. Seventy-seven scaffolds $(52.5 \mathrm{Mb}$, ranging from $1.5-5565 \mathrm{~kb}$ ) had no heterozygous genotypes in the female but a minimum of one heterozygous genotype in the male and ten of those scaffolds $(10.1 \mathrm{Mb})$ mapped to the Z-chromosome in the golden eagle genome. Aside the larger fraction of the $Z$ scaffolds which had no variation on $\mathrm{Z}$, about $62 \%$ of the $\mathrm{Z}$-chromosome in the female had also considerably fewer heterozygous sites than the 
Table 1 Information about heterozygosity for a female and male. Heterozygosity for each of the male and female for scaffolds that map to the $A$ and $Z$ in the golden eagle genome with known chromosomes. Numbers of heterozygous (hets.) sites, scaffolds and windows of size 50,000 bases. Total number of scaffolds and $50 \mathrm{k}$ windows were 1141 and 23,585 respectively

\begin{tabular}{|c|c|c|c|c|}
\hline & Female Z & Female A & Male Z & Male A \\
\hline Proportion of heterozygous sites before filtering & 0.00534 & 0.00067 & 0.00050 & 0.00065 \\
\hline Proportion of heterozygous sites after filtering & 0.00010 & 0.00018 & 0.00007 & 0.00019 \\
\hline Scaffolds with no heterozygous sites & $134(80 \%)$ & $720(74 \%)$ & $130(77 \%)$ & $712(73 \%)$ \\
\hline Size of scaffolds with no heterozygous sites (kb) & $26,625(31 \%)$ & $55,018(5 \%)$ & $20,010(23 \%)$ & $65,907(6 \%)$ \\
\hline Scaffolds with heterozygous sites & 34 & 254 & 38 & 262 \\
\hline Heterozygous sites per window (50 kb) (median) & 0 & 1 & 0 & 2 \\
\hline Standard deviation per window (50 kb) & 43.0 & 12.3 & 8.1 & 12.2 \\
\hline Coefficient of dispersion (CD) & 360.6 & 16.1 & 20.2 & 15.6 \\
\hline Windows with no heterozygous site & 1398 & 10,264 & 1267 & 9857 \\
\hline Windows with heterozygous sites & 304 & 11,619 & 435 & 12,026 \\
\hline
\end{tabular}

male (supplement Fig. 3), but some show autosomal levels of heterozygosity in the female (separately marked in Fig. 2A). Four of these scaffolds also exhibited autosomal levels of depth in the female (Fig. 1) and two of those scaffolds ("NW_011950951.1" "NW_011950990.1") in the female had the highest number of heterozygous sites (1823, 5568), followed by NW_011951047.1 which had 450 sites.

The four $\mathrm{Z}$ chromosomal scaffolds that had a malelike pattern of autosomal depth and heterozygosity in the female were further analysed in windows of $50 \mathrm{~Kb}$, as heterozygous sites can be restricted to small parts of the scaffold (Fig. S6). An examination of the number of filtered heterozygous sites per $50 \mathrm{~Kb}$ window in these four scaffolds in the female, showed that NW_011950951.1, NW_011950990.1 consisted of either 1 or 2 continuous regions, whereas the other two were more fragmented.

The average heterozygosity per scaffold, prior to filtering, was $>10$-fold higher in the female than the male for the Z-chromosome (Table 1), and several scaffolds were even higher (Fig. 2B). The filtering removed most of this excess heterozygosity in the female (Fig. 2C, D and E). As the pattern of excess heterozygosity in the female was primarily seen in $\mathrm{Z}$ rather than autosomal scaffolds, we postulate that these instances might represent the mapping of diverged homologous reads from the $\mathrm{W}$ chromosome.

Overall, the distributions of heterozygous sites per window was similar for the male and the female and almost half of the windows had no heterozygosity (49\% in the female and $47 \%$ in the male). When the windows were grouped by $\mathrm{Z}$ and autosomes, a difference between the sexes was observed for the Z-chromosome (Table 1 and Fig. S4 and S5). As expected, there was a higher proportion of windows on $\mathrm{Z}$ with no heterozygous sites in the female $(82 \%)$ than in the male $(74 \%)\left(P=6.111 * 10^{-8}\right.$, Fishers exact test). However, the 10 most variable
$50 \mathrm{~kb}$ windows in the female, with rate of heterozygous sites ranging from $0.17-1.73 \%$ all came from the scaffold NW_011950990.1 which map to Z. The window in the male with the largest rate of heterozygous sites had $0.15 \%$. This difference in the distribution of heterozygosity per $50 \mathrm{~kb}$ windows on the $\mathrm{Z}$ chromosome per sex is also reflected in the average number and standard deviation of heterozygous genotypes per window, which was larger in the female $Z$ (5.1 and 43) than in the male Z (3.2 and 8.1), whereas no differences were observed in these descriptive statistics for the autosomes. This means that the distribution of heterozygous genotypes was more clumped for $\mathrm{Z}$ in the female (Coefficient of dispersion, $\mathrm{CD}=360.5)$ than in the male (20.2) and the autosomes of both sexes $(\sim 16)$ (Table 1$)$.

\section{Mapping}

Mapping the 1141 scaffolds from the golden eagle scaffold assembly to the chicken genome, using LASTZ, resulted in 110 scaffolds $(86.5 \mathrm{Mb})$ correctly assigned to the Z-chromosome, and 940 scaffolds correctly assigned to autosomes, according to the comparison of mapping to the golden eagle chromosome-assembled genome. On the other hand, 33 scaffolds $(0.59 \mathrm{Mb}$, amounting to $0.69 \%$ of the total length of scaffolds) were wrongly assigned to the Z-chromosome, and 58 scaffolds $(0.27 \mathrm{Mb}, 0.024 \%)$ were wrongly assigned to autosomes (Table 2).

\section{PCA}

The analysis of the loadings of 164,952 SNPs from the PCA analysis (Fig. S1), based on 133 RADseq individuals with an average sequencing depth per site of 2.25 per individual, was limited to the 280 scaffolds ( $40 \mathrm{Z}$ and 240 autosomal) that had more than 50 SNPs (accounting for 98.3\% of the genome). We calculated the $95 \%$ range of SNP-loadings on PC1 (i.e., the quantiles 0.025 and 0.975) 


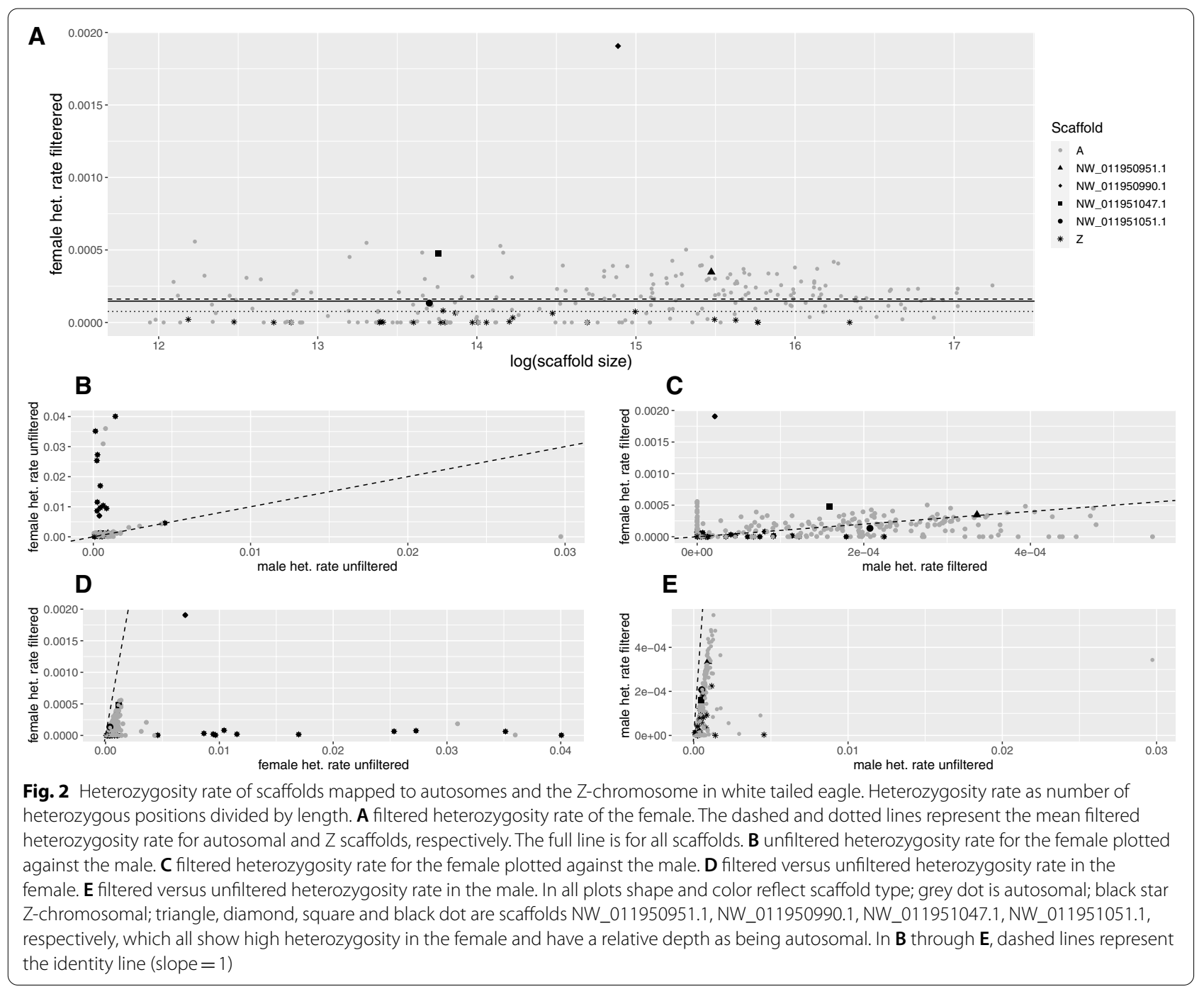

in our attempt to identify scaffolds belonging to the Z, using a threshold (0.1006) that corresponds to 3 standard deviations above the mean (Fig. 3A and B, Table 2). Of the scaffolds included in this analysis, 28 (78\%) scaffolds from the Z-chromosome were above this threshold, accounting for $69.3 \mathrm{Mb}(83.6 \%$ of the total length of $\mathrm{Z}$ scaffolds used in this analysis). In contrast, only 9 (3.75\%) of the autosomal scaffolds were above the threshold, amounting to $11.7 \mathrm{Mb}(1.1 \%$ of the total length of autosomal scaffolds used in this analysis). Thus, the range of PC1 loadings provides some discriminatory power to distinguish $\mathrm{Z}$ from autosomal scaffolds.

\section{Comparison of the four methods}

Using chromosome assignments obtained by mapping the golden eagle scaffold assembly to the golden eagle genome with assigned chromosomes, the most successful method was mapping to the chicken genome, finding
$98.12 \%$ of the expected size (Table 2, Fig. 4). In second place was the depth analysis with $86.41 \%$ and, in third, the SNP-loading with $78.61 \%$. Heterozygosity was poorly suited to find Z-chromosomal scaffolds as a large fraction of scaffolds had no variation, and some Z-chromosomal scaffolds were found to be highly variable in the female (likely due to the mapping of reads that belong to the $\mathrm{W}$ chromosome). Depth, mapping to the chicken and SNPloading all found false positives, i.e., autosomal scaffolds that were categorised as Z-chromosomal scaffolds $(0.09$, 0.59 and $11.72 \mathrm{Mb}$, respectively). All approaches resulted in false negatives i.e., Z-chromosomal scaffolds categorised as autosomal (Table 2), but least with mapping to the chicken $(0.27 \mathrm{Mb})$, whereas depth, heterozygosity, and SNP-loading had 10.05, 60.21 and 13.64 Mb of false negatives, respectively. Forty-five very short Z-chromosomal scaffolds (with a total length of $0.22 \mathrm{Mb}$ ) were not found by any analysis, and were only found when 
Table 2 Classification of scaffolds identified as Z or autosomal scaffolds. Classification for each of the approaches: depth, heterozygosity, LASTZ, and SNP-loading analysis. The identification was found by comparison to the golden eagle genome bAquChr1.2 (GCA_900496995.2) with known chromosomes. Results for the different methods are given in a) for total size of scaffolds (bp), and in b) for the number of scaffolds, missing is obtained by comparison with the golden eagle scaffold assembly

\begin{tabular}{|c|c|c|c|c|c|c|c|c|c|}
\hline \multirow[b]{2}{*}{ a) } & & \multicolumn{2}{|l|}{ Depth } & \multicolumn{2}{|c|}{ Heterozygosity } & \multicolumn{2}{|l|}{ LASTZ } & \multicolumn{2}{|c|}{ SNP-loading } \\
\hline & & Z & $A$ & $Z^{a}$ & $A$ & Z & A & Z & A \\
\hline & Z & $76,239,124$ & $10,056,095$ & - & $60,214,856$ & $86,569,008$ & 270,522 & $69,355,267$ & $13,642,226$ \\
\hline & A & 93,786 & $1100,765,118$ & - & $1,050,885,219$ & 597,603 & $1,105,305,943$ & $11,720,756$ & $1,078,283,284$ \\
\hline & Total & & $1,187,154,123$ & & $1,159,757,217$ & & $1192,725,744$ & & $1,173,001,533$ \\
\hline & Missing & & $5,571,621$ & & $29,104,198$ & & 0 & & $19,714,211$ \\
\hline \multirow[t]{4}{*}{ b) } & Z & 79 & 13 & - & 34 & 110 & 58 & 28 & 12 \\
\hline & A & 14 & 512 & - & 254 & 33 & 941 & 9 & 231 \\
\hline & Total & & 618 & & 365 & & 1141 & & 280 \\
\hline & \#NA & & 523 & & 776 & & 0 & & 861 \\
\hline
\end{tabular}

${ }^{a}$ values not assigned due to lack of heterozygosity on the $Z$ chromosome

the golden eagle scaffold assembly was mapped to the golden eagle with known chromosomes. Mapping of the golden eagle scaffold assembly to the golden eagle with assembled chromosomes revealed $98.42 \%$ of the whole known Z-chromosome (Table 2, Fig. 4). Though the goal of the study was to evaluate the approaches separately, a combined analysis (Fig. 4) where at least two of three approaches (e.g., depth, mapping to the chicken, and SNP-loading) were compared, detected between $75.29-86.29 \%$ of the size of the Z-chromosome of the golden eagle genome, and only the approach combining depth and mapping to the chicken found false positives, which was less than $<0.01 \%$ of the size of the golden eagle Z-chromosome.

\section{Discussion}

Three of the four methods evaluated in this study; the relative depth, mapping to chicken, and SNP-loadings, were able to detect a high fraction of the Z-chromosome of the white-tailed eagle that had been mapped on the golden eagle scaffold assembly. The success of the methods varied as they may be affected differently by the small population size of the study species. The approaches applying heterozygosity and PCA are expected to be more affected by a small population because they analyse genomic and population diversity, whereas depth and mapping are expected to be less affected by the low diversity in a small population.

The mapping of contigs to genome sequences from a distantly related species such as golden eagle to chicken can be problematic due to architectural changes such as translocations and inversions. Minor mismatches, e.g., transposable elements and mutations, may further impact the success of finding the Z-chromosome. However, sex chromosomes may be well preserved in birds e.g., $\mathrm{Xu}$ and Zhou [19], and this effect seems to be mini$\mathrm{mal}$ in the case of mapping the golden eagle scaffold assembly to the chicken with a split time $>80$ million years [5].

The $\mathrm{Z}$ scaffolds that were not detected using the SNPloading approach are likely due to parts of the Z-chromosome that lack variation, or that share homologous regions in the distinct sex chromosomes and do not contribute to the difference between the sexes in the PCA-plot. The PCA approach found few false positives, possibly due to the lack of a precise distinction between the range of loadings observed for the autosomal and Z-chromosomal scaffolds. Considering the information from the mapping it is clear that the Z-scaffolds have higher impact, as most false positives were just above the threshold of three SDs (i.e., 0.10 95\% SNP loading range), and only two autosomal scaffolds were larger than $\sim 0.11$ comprising only a total size of $1.73 \mathrm{Mb}$, or $14 \%$ of the false positives. The SNP-loading approach also found false negatives (Table 2) and we feel this deserves further research.

Here, the approach of looking at all scaffolds in a single PCA was used, but this could potentially be optimized by using sliding windows [41] to identify signals different from the overall population signal. However, this also requires diversity on the homogametic sex chromosome in males compared to females, which may be lacking in small populations such as in the Icelandic white-tailed eagle.

Inspection of the heterozygosity for all scaffolds revealed that it is difficult to distinguish between autosomal and Z-chromosomal scaffolds without any prior knowledge. However, there was a difference in the average heterozygosity between autosomal and Z-chromosomal scaffolds, especially in the female. Small 


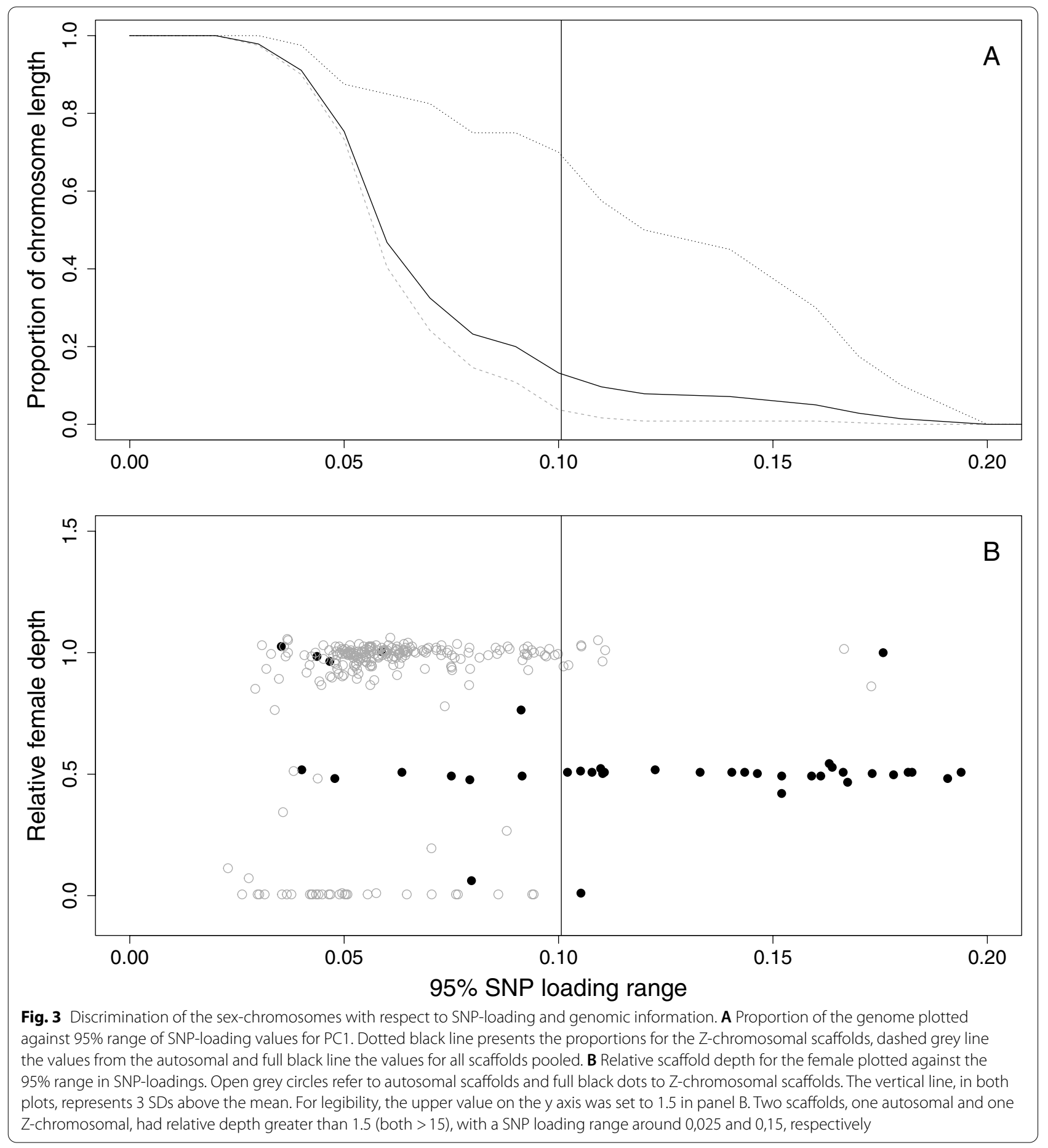

populations, such as the white-tailed eagles in Iceland [9], have reduced heterozygosity and long runs of homozygosity were observed on the Z-chromosome and the autosomes, making it more difficult to distinguish among the chromosomal types. Furthermore, there is a clear overlap in scaffolds with some heterozygosity which might belong to PAR and non-homologus regions, e.g., due to inversions, on the Z- and W-chromosomes. PAR and the nonrecombining homologous regions, could explain some deviations in the prediction of the Z-chromosome in the SNP-loading analysis but these regions are probably small, and thus won't display the signal of an 


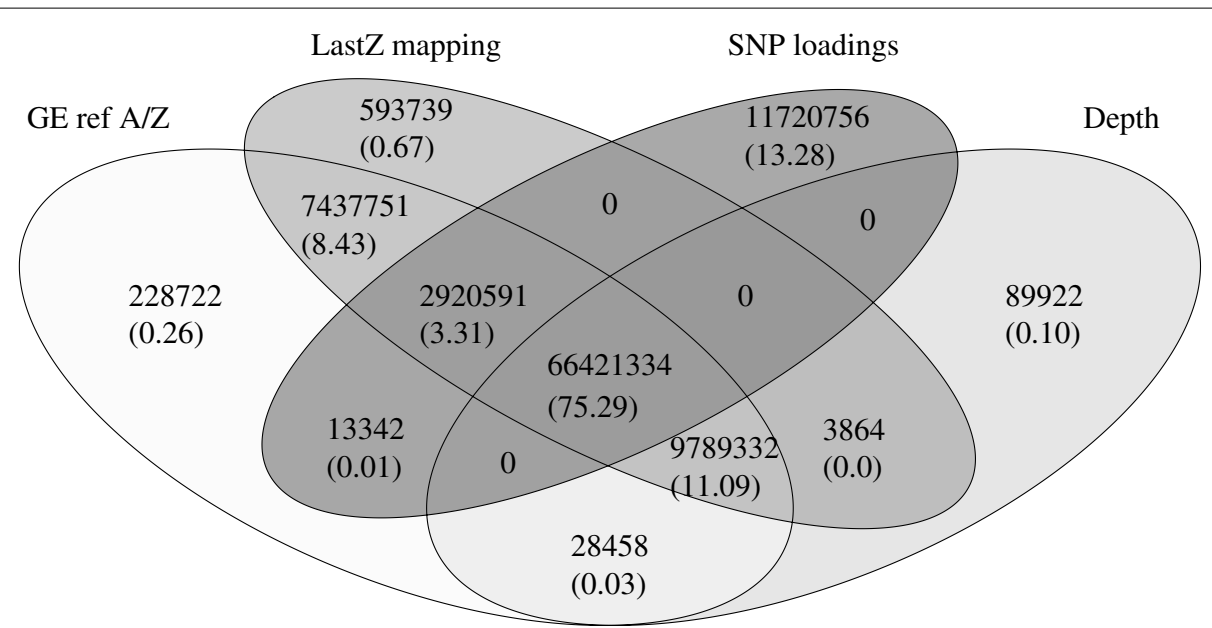

Fig. 4 Venn diagram summarizing the size of scaffolds in bases identified as Z-chromosome with the three different analyses: mapping, depth and SNP-loadings. The Z-chromosomal scaffolds were assigned by mapping the genome with scaffolds to the genome with known chromosomes. Values in parentheses represent percentage size compared to the size of the known Z-chromosome. Notice that the percentage found by mapping the golden eagle scaffold assembly to the golden eagle genome is only $98.42 \%$

autosome in the depth analysis. Although genome wide information from a single individual can provide assessment of variation within populations, it can be biased due to missing chromosomal fragments and thus the overall success of the method. However, the two high depth individuals here show no clear indication of such deviation, as we obtain most of the Z-chromosome in the analysis.

The relative depth analysis revealed $86.41 \%$ of the expected size of the Z-chromosome and found few false positives. Four scaffolds were noted as false negatives in one of the two depth analysis. These four scaffolds (NW_011950951.1, NW_011950990.1, NW_011951047.1, and NW_011951051.1) make up about $10 \mathrm{Mb}$ and show the highest heterozygosity of all Z-chromosomal scaffolds after filtering; their levels are comparable or even higher than observed for the autosomal scaffolds. Three of the four scaffolds showed low 95\% SNP-loading ranges (all around 0.05), unlike the scaffolds contributing to the separation of the sexes. One scaffold (NW_011950990.1) had a very high 95\% SNPloading range and very high heterozygosity. This signal in these four $\mathrm{Z}$ scaffolds, and position at the end of the Z-chromosome supports that they belong to the pseudoautosomal regions (PAR) as seen in other birds [29, 42]. In birds, PAR vary greatly in size from just a few $\mathrm{Mb}$ to more than $60 \mathrm{Mb}$ [29]. Alternatively, they could represent non-recombining homologous regions (gametologs) [19, 43] which can be expected to have even higher heterozygosity in females than within the recombining Z-chromosomes in the homogametic males or the autosomes, because such regions could have evolved independently for millions of years. Two of the four scaffolds mentioned above, NW_011950990.1 and NW_011951051.1, display a higher heterozygosity ratio in the female compared to the male (17, and 2.5 times higher, respectively), as expected for gametologous regions, whereas the other two NW_011950951.1, and NW_011951047.1, may present PARs, as they display a ratio close to one between the sexes (1.08 and 0.78 , respectively). A fully annotated genome of the white-tailed eagle would provide further information about these gametologous regions within the Z- and W-chromosome.

Although depth analysis has shown to be a promising method to identify sex chromosomes [23, 44], it is not error free. Scaffolds belonging to the Z-chromosome can have a depth as high as autosomes, as variance in depth can be large in small scaffolds which may be poorly sampled due to low variation, or the scaffolds include regions from both Z- and W-chromosomes i.e., gametologs and the PAR regions. Here the best approach for identifying the homogametic sex chromosome was mapping to a reference with annotated homogametic sex chromosome. To identify the Z-chromosome, a combination of the mapping with at least one other analysis is recommended as it may result in fewer potential false positives and negatives. Further, it should be noted that the methods used here maybe more applicable in taxa with relative stable sex chromosomes, such as mammals and birds $[19,20]$, but less effective in taxa such as fish where the sex chromosomes can be less differentiated [21,22].

The dynamic nature of the Z-chromosome (e.g., songbirds $[36,45])$ and potential deviations in synteny may introduce errors into assemblies of two species, however, there is significant and relevant justification for doing so. 
The approach using a different reference from the study species has successfully been employed in other studies [2-4]. Mapping of our novel white-tailed sequences against the golden eagle assemblies, one assembled to scaffolds, and second assembled to chromosomes, made it possible to evaluate the precision of these approaches to a greater extent. This study highlights potential problems when trying to identify the homogametic sex chromosome that are specific to small populations, which bears importance for the conservation of species at risk.

Even though all known eukaryote species may soon be sequenced [46], it will still be a long time before all parts of their chromosomes have been identified. Thus, it is important to further explore these different methods and how they depend on sequence variation and scaffold sizes, as variation in the different chromosomes will differ due to different effective population sizes and evolutionary histories.

\section{Conclusion}

The best performing approach for identifying the homogametic sex chromosome in the small population of white-tailed eagle was obtained by aligning of the reference genome to a species with annotated sex chromosomes. The second-best approach was an analysis of read depth per scaffold, and third was an analysis of SNP-loadings in a PCA. Identification using genomic diversity approaches. The utility of the SNP-loadings and heterozygotic differences between the sexes suffers likely by the small population size and a recent population bottleneck in the study populations. Evaluation of these methods are highly relevant as genomic regions vary in effective population size and can have different evolutionary histories.

\section{Methods}

\section{Sample collection, laboratory work and sequencing}

Blood samples were collected from white-tailed eagle chicks as a part of an ongoing monitoring program in Iceland since 2001 by the Natural History Institute of Iceland. The sex of the chicks was determined in the field based on tarsus thickness and weight [47]. Three to ten $\mathrm{mL}$ of blood was extracted from each chick. The blood was stored in EDTA buffer at -20 degrees Celsius until DNA extraction.

DNA from blood samples of 135 chicks was extracted using the ThermoFisher GeneJET Whole Blood Genomics DNA Purification Mini Kit following the standard protocol [48]. DNA concentration was estimated using the NanoDrop 1000 and run on $0.7 \%$ agarose gels to evaluate the fragment size. Samples with concentration higher than $60 \mathrm{ng} / \mu \mathrm{l}$ were selected for library preparation and sequencing. The 133 of 135 extracts were double digest restriction-site associated DNA sequenced (RADseq) on the Illumina HiSeq2500 (see supplementary text 1 for full description).

A male and female white-tailed eagle were selected for high-depth whole genome shotgun sequencing with two lanes each on an Illumina HiSeqX. Library preparation and sequencing was done at deCODE genetics, using the TruSeq Nano sample preparation method [49].

Two reference assemblies from male golden eagles (ZZ), one in 1142 scaffolds and one assembled to chromosome level (GenBank Assembly Accession numbers: GCA_000766835.1 and GCA_900496995.2, respectively), and a female chicken assembly (ZW) (GenBank Assembly Accession: GCA_000002315.3) were downloaded from NCBI and used in the analysis $[12,50]$.

\section{Sequence cleaning and mapping}

The white-tailed eagle RADseq data was demultiplexed, sorting sequence reads into individual files, both for forward and reverse sequences using the command 'process_radtags' in Stacks version 1.47 [51, 52]. Default settings were used for the RADseq data, applying the option " $r$ " to rescue barcodes and RAD-tags.

After demultiplexing, FastQC [53] was run for quality control. For the RADseq data, an excess of specific sequences (kmers) were removed using AdapterRemoval v2 (version 2.2.2) [54]. The high depth shotgun sequenced individuals were tested in the same way but found no excess of kmers.

The Burrows-Wheeler Aligner (BWA) mem and SAMtools version $0.7 .17-$ r1188 and 1.7 , respectively $[55,56]$ were used to process RADseq and high depth shotgun data and map reads to the golden eagle scaffold assembly of 1142 scaffolds with no identified chromosomes (GCA_000766835.1) [12] using default settings in both instances.

\section{Four different approaches to find the Z-chromosome - depth, Heterozygosity, mapping and SNP-loadings} Four different approaches were used to identify scaffolds in the white-tailed eagle genome belonging to the Z-chromosome, by comparison with the golden eagle scaffold assembly with no chromosomes (GCA_000766835.1). An assembly consisting of 1141 assembled scaffolds, excluding mtDNA, and a total of $1192,725,744 \mathrm{bp}$, ranging in size from 913 to $30,727,332 \mathrm{bp}$ with a median of $5587 \mathrm{bp}$, and average length of 1,045,334bp (SD 3,203,066 bp). An overview of the methods is presented in Fig. 5 and the data used in each analysis is available in supplementary Table S1.

\section{Depth}

For the high-depth white-tailed eagle sequencing data, the average autosomal sequencing depth was estimated 


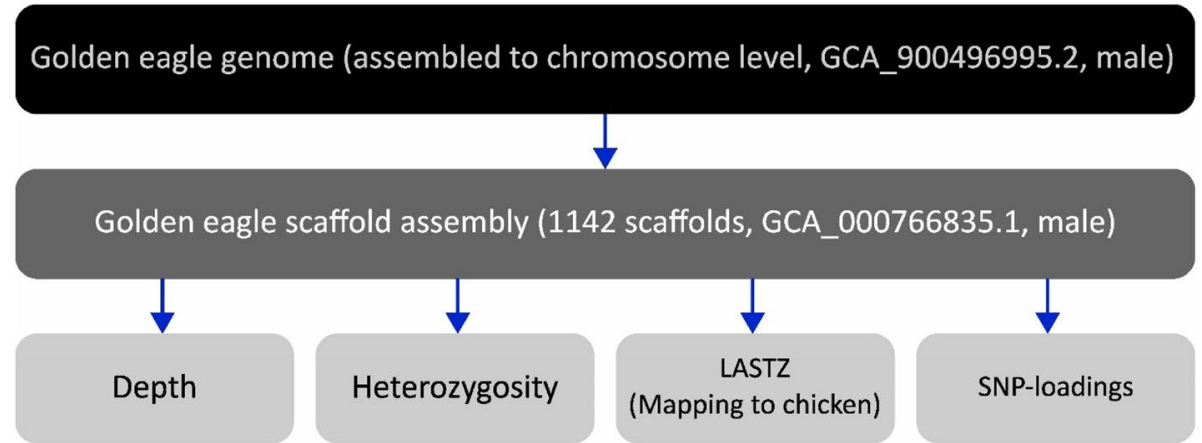

Fig. 5 Schematic overview of the methods used to identify the Z-chromosome in a scaffold assembled genome. The golden eagle genome referred to in the dark grey box represents the reference in which we are attempting to identify scaffolds belonging to the Z-chromosome. The golden eagle genome in the black bar is the genome with known chromosomes, used to identify which scaffolds in the dark grey boxed genome probably belong to Z-chromosome (and autosomes) - to use as a reference. The light grey boxes are the four approaches we tested to find the scaffolds belonging to the Z-chromosome: 1) Depth: analysis of difference in sequencing depth between scaffolds in a high depth whole genome sequenced white-tailed eagle female. 2) Heterozygosity: analysis of the difference in heterozygosity per scaffold a high depth whole genome sequenced white-tailed eagle male and female. 3) LASTZ: mapping of the golden eagle reference genome to the chicken genome using LASTZ. 4) SNP-loadings: analysis of SNP-loadings for principal components splitting the sexes, in 133 RADseq white-tailed eagle individuals

for the male and female separately, as the mode of the number of mapped reads per position across all scaffolds based on results from the command "bedtools coverage" from Bedtools v2.18.2 [57]. Using these averages, 195 for the female and 181 for the male, the relative sequencing depth was calculated for each position in each scaffold for both individuals. The per-scaffold relative sequence depth was then estimated for the female and male separately, as the mode across positions. Positions in autosomal scaffolds are expected to have a relative depth of 1 in both sexes, whereas Z-chromosomal scaffolds are expected to have a relative depth of 0.5 in females and 1 in males. As the estimate of relative depth may be less reliable for smaller scaffolds, the dependency of the relative mode depth due to scaffold size was analysed by calculating the variance in the depths per interval of scaffold sizes, transformed to a log scale. The distribution of the proportions of scaffolds at each interval was summarized with a cumulative percentage curve. In addition, the depth per scaffold was evaluated by comparing the per-scaffold relative sequencing depth between the two individuals: male over female. Scaffolds with a relative sequencing depth below 0.25 and above 1.5 were removed (corresponding to 523 scaffolds, and $0.47 \%$ of the genome). This ratio is expected to be around two for Z-chromosomal scaffolds and one for the autosomal scaffolds, as the male has two copies of $\mathrm{Z}$ and the female one. Thus, a cut-off was set at 1.5 .

\section{Heterozygosity}

Sex differences in heterozygosity were assessed by comparing numbers of heterozygous sites per scaffold based on genotypes of the high-depth white-tailed eagle male and female, called using Graphtyper [58, 59] with default settings. The variation on the Z-chromosome is expected to be $3 / 4$ of the autosomes and it should be restricted to the male, except for the PAR and non-recombining homologous regions. As scaffolds vary in length and may include short variable regions, the variation was also analysed per $50 \mathrm{~kb}$ window. Genotypes were filtered for quality using vcftools and bcftools version 0.1 .15 and 1.7 , respectively $[60,61]$ before counting, using minimum GQ score 20, minimum Q score 1000, missingness 1 (both individuals had to have a valid genotype at the site), mapping quality equal to $60(\mathrm{MQ})$, and only biallelic sites. Two additional criteria were applied to remove sites with likely spurious heterozygous genotypes. First, heterozygous genotypes where the number of mapped reads deviated significantly from the mode depth of the scaffold, based on a two-sided Poisson test $(P<0.01)$ were excluded. Second, we used a binomial test to assess whether the proportion of reads in heterozygous genotypes, either in the male or the female, deviated from the 50/50 expectation, using $P<0.05$ as the exclusion threshold.

\section{Mapping}

In order to assign the short reads from the white-tailed eagle to chromosomes, the 1142 scaffolds from the golden eagle scaffold assembly (which the white-tailed eagle genome had been mapped on) were mapped to the chicken genome, which has assigned chromosomes, using LASTZ [30]. Standard settings were used with the following modifications: ambiguous=iupac, gfextend, chain, gapped. Scaffolds in the golden eagle which 
mapped better to the Z-chromosome than any other chromosome, measured as most bases mapped, were deemed to belong to the golden eagle Z-chromosome.

\section{SNP-loadings}

A PCA analysis of 133 low-depth RAD sequenced whitetailed eagle individuals was constructed using PCangsd version 1.0 [62], an extension of ANGSD [63], as described below. A clear split between males and females was observed along the first principal component (PC) (Fig. S1). Loadings obtained with PCangsd were used to identify which parts of the scaffolds induced the split, with the "-selection" option [62] and with sites passing the following filters: a minimum $25 \%$ of individuals had to have valid genotypes, only unique mapping sites, base quality minimum 20 , mapping quality minimum 30 , SNP $p$-value 1e-6. ANGSD uses genotype likelihoods to tackle the restrictions of low depth [63, 64]. To assess which scaffolds contributed to the split on the first axis (PC1), a 95\% range of loading values for all SNPs per scaffold was calculated using R [65] and compared between scaffolds with more than 50 SNPs. The distributions of the range of loading values were summarized with accumulation curves, combined for all scaffolds, and separately based on the results obtained by the mapping on the autosomes and $\mathrm{Z}$ chromosome. Scaffolds were assigned to the Z-chromosome or autosomes depending on whether the range-values were above or below a threshold of three standard deviations from the mean (covering 99\% of a normally distributed variable).

\section{Comparison of the four methods}

To evaluate how well the four approaches performed, the golden eagle scaffold assembly (GCA_000766835.1) was mapped to a golden eagle genome with known chromosomes (GCA_900496995.2) using LASTZ with the same settings and cut-off as described previously. In the results, the outcome of this mapping was used as the true chromosome identity of the 1141 scaffolds that was used to assess the accuracy of our four different approaches to identify $\mathrm{Z}$ chromosome scaffolds (Fig. 5 and Table 2). A total of 168 scaffolds were assigned to the Z-chromosome, with a total length of $86,839,530 \mathrm{bp}$ $($ mean $=516,902$, $s d=1,509,132$, and median $=5236)$, which is slightly smaller than the Z-chromosome in the newly released genome of 88,216,475bp (GenBank Assembly Accession: GCA_900496995.2). The autosomal loci mapped to 973 scaffolds of a size of $1,105,886,214 \mathrm{bp}$ $($ mean $=1,136,574$, sd $=3,403,676$, and median $=5674)$. The overlap of these four methods was summarized with the R-package VennDiagram [66].

Summary of the data and further statistical analyses, if otherwise not stated was done using $\mathrm{R}$.

\section{Supplementary Information}

The online version contains supplementary material available at https://doi. org/10.1186/s12864-022-08393-z.

Additional file 1: Supplementary figures S1-S6 and supplementary text 1 describing DNA sequencing and extraction.

Additional file 2: Table S1. Full raw data file, containing all values used in the calculations.

\section{Acknowledgements}

Thanks to Gunnar T. Hallgrimsson from Department of Life and Environmental Sciences, University of Iceland, Menja von Schmalensee and Robert A.

Stefansson from West-Iceland Centre of Natural History, and Kristinn Haukur Skarphédinsson from the Icelandic Institute of Natural History for sample collection.

Thanks to Jonas Meisner from section for Computational and RNA Biology, University of Copenhagen, for great help with PCAngsd.

Thanks to Agnar Helgason from deCODE genetics for guidance in analysis, writing, and access to facilities at deCODE genetics. Further, thanks to deCODE genetics for sequencing of the two white-tailed genomes.

\section{Authors' contributions}

$\mathrm{CCRH}$ and SP designed the study; KMW prepared the RADseq libraries; $\mathrm{CCRH}$ and SP analyzed the data; CCRH, KMW and SP wrote the paper. All authors read and approved the final manuscript.

\section{Funding}

The study was supported by Research Grant nr 185280-052 from The Icelandic Research Council, the Doctoral student fund of the University of Iceland and The University of Iceland Research fund.

\section{Availability of data and materials}

The raw dataset supporting the conclusions of this article is available in the DRYAD data repository https://doi.org/10.5061/dryad.v9s4mw6vs.

Further, the analysed dataset supporting the conclusions of this article is included in the supplementary.

\section{Declarations}

\section{Ethics approval and consent to participate}

The DNA sequences analysed in this study were obtained from an existing database generated from a study on genomic variation in white-tailed eagles [67], (Hansen CCR, Rasmussen JA, Ballesteros JAC, Sinding MHS, Hallgrimsson GT, Stefansson RA, et al: Genomics of white-tailed eagle (Haliaeetus albicilla) in the North-Atlantic islands reveal low diversity and substantial inbreeding in comparison with the mainland populations. Submitted). The DNA was extracted from blood samples sampled from chicks in an ongoing monitoring project of the species in Iceland, led by Kristinn H. Skarphéðinsson a specialist at the Icelandic Institute of Natural History (IINH). At the time of sampling, of the DNA analysed, 2000-2011, there were no ethical committee who handled sampling from wild animals in Iceland. The research and sampling were conducted with permission from the IINH, for tagging and approaching nests of rare birds. The sampling of blood was taken by trained experts which took care in not injuring the chicks of this endangered species. The overall work of the genomic study is in accordance with the ARRIVE guidelines.

\section{Consent for publication \\ Not applicable.}

\section{Competing interests}

The authors declare no competing interests.

\section{Author details}

${ }^{1}$ Department of Life and Environmental Sciences, University of Iceland, Reykjavik, Iceland. ${ }^{2}$ Current: Fisheries and Oceans Canada, Pacific Biological Station, Nanaimo, BC, Canada. 
Received: 10 September 2021 Accepted: 15 February 2022

Published online: 24 February 2022

\section{References}

1. Hill WG, Robertson A. The effect of linkage on limits to artificial selection. Genet Res. 1966;8:269-94.

2. de Manuel M, Barnett R, Sandoval-Velasco M, Yamaguchi N, Garrett Vieira F, Zepeda Mendoza ML, et al. The evolutionary history of extinct and living lions. Proc Natl Acad Sci. 2020;117:10927-34.

3. Pedersen CET, Albrechtsen A, Etter PD, Johnson EA, Orlando L, Chikhi L, et al. A southern African origin and cryptic structure in the highly mobile plains zebra. Nat Ecol Evol. 2018;2:491-8.

4. Pečnerová P, Garcia-Erill G, Liu X, Nursyifa C, Waples RK, Santander CG, et al. High genetic diversity and low differentiation reflect the ecological versatility of the African leopard. Curr Biol. 2021. https://doi.org/10. 1016/j.cub.2021.01.064.

5. Jarvis ED, Mirarab S, Aberer AJ, Li B, Houde P, Li C, et al. Phylogenomic analyses data of the avian phylogenomics project. GigaScience. 2015:4:1-9.

6. Boulesteix M, Weiss M, Biémont C. Differences in genome size between closely related species: the Drosophila melanogaster species subgroup. Mol Biol Evol. 2006;23:162-7.

7. Ellegren H. Evolutionary stasis: the stable chromosomes of birds. Trends Ecol Evol. 2010;25:283-91.

8. Zhang G, Li C, Li Q, Li BB, Larkin DM, Lee C, et al. Comparative genomics reveals insights into avian genome evolution and adaptation. Science. 2014:346:1311-20.

9. Skarphéðinsson KH. Haförninn. Reykjavik: Fuglavernd (Fuglaverndarfélag íslands); 2013.

10. BirdLife International. 2016. Aquila chrysaetos. The IUCN Red List of Threatened Species. 2016:e.T22696060A93541662. http://dx.doi.org/ 10.2305/IUCN.UK.2016-3.RLTS.T22696060A93541662.en.

11. BirdLife International. 2016. Haliaeetus albicilla. The IUCN Red List of Threatened Species. 2016:e.T22695137A93491570. http://dx.doi.org/ 10.2305/IUCN.UK.2016-3.RLTS.T22695137A93491570.en.

12. Doyle JM, Katzner TE, Bloom PH, Ji Y, Wijayawardena BK, DeWoody JA. The genome sequence of a widespread apex predator, the golden eagle (Aquila chrysaetos). PLoS One. 2014;9:20-2.

13. Kim JA, Kang SG, Jeon HS, Jeon JH, Jang JH, Kim S, et al. Complete mitogenomes of two Accipitridae, Haliaeetus albicilla, and Pernis ptilorhynchus. Mitochondrial DNA Part B: Resources. 2019;4:392-3.

14. Damas J, O'Connor R, Farré M, Lenis VPE, Martell HJ, Mandawala A, et al. Upgrading short-read animal genome assemblies to chromosome level using comparative genomics and a universal probe set. Genome Res. 2017:27:875-84.

15. Warren WC, Clayton DF, Ellegren H, Arnold AP, Hillier LW, Künstner A, et al. The genome of a songbird. Nature. 2010;464:757-62.

16. Sigeman H, Ponnikas S, Chauhan P, Dierickx E, M de L Brooke, Hansson B. Repeated sex chromosome evolution in vertebrates supported by expanded avian sex chromosomes. Proc R Soc B. 2019;286:20192051.

17. Bellott DW, Skaletsky H, Pyntikova T, Mardis ER, Graves T, Kremitzki C, et al. Convergent evolution of chicken $Z$ and human $X$ chromosomes by expansion and gene acquisition. Nature. 2010;466:612-6.

18. Palmer DH, Rogers TF, Dean R, Wright AE. How to identify sex chromosomes and their turnover. Mol Ecol. 2019;00:1-16.

19. Xu L, Zhou Q. The female-specific W chromosomes of birds have conserved gene contents but are not feminized. Genes. 2020;11:1-14

20. Graves JAM. Sex chromosome specialization and degeneration in mammals. Cell. 2006;124:901-14.

21. Kitano J, Peichel CL. Turnover of sex chromosomes and speciation in fishes. Environ Biol Fish. 2012:94:549-58.

22. Kikuchi K, Hamaguchi S. Novel sex-determining genes in fish and sex chromosome evolution. Dev Dyn. 2013;242:339-53.

23. Nadachowska-Brzyska K, Burri R, Ellegren H. Footprints of adaptive evolution revealed by whole Z chromosomes haplotypes in flycatchers. Mol Ecol. 2019;28:2290-304.

24. Webster TH, Couse M, Grande BM, Karlins E, Phung TN, Richmond PA, et al. Identifying, understanding, and correcting technical artifacts on the sex chromosomes in next-generation sequencing data. GigaScience. 2019;8:1-11.
25. Feron $\mathrm{R}$, Pan $\mathrm{Q}$, Wen $\mathrm{M}$, Imarazene $\mathrm{B}$, Jouanno $\mathrm{E}$, Anderson J, et al. RADSex: a computational workflow to study sex determination using restriction site-associated DNA sequencing data. Mol Ecol Resour. $2021 ; 21: 1715-31$

26. Rangavittal S, Stopa N, Tomaszkiewicz M, Sahlin K, Makova KD, Medvedev P. DiscoverY: a classifier for identifying $Y$ chromosome sequences in male assemblies. BMC Genomics. 2019:20:641.

27. Nursyifa C, Brüniche-Olsen A, Garcia Erill G, Heller R, Albrechtsen A. Joint identification of sex and sex-linked scaffolds in non-model organisms using low depth sequencing data. Mol Ecol Resour. 2021;22:458-67.

28. Brelsford A, Lavanchy G, Sermier R, Rausch A, Perrin N. Identifying homomorphic sex chromosomes from wild-caught adults with limited genomic resources. Mol Ecol Resour. 2017;17:752-9.

29. Zhou Q, Zhang J, Bachtrog D, An N, Huang Q, Jarvis ED, et al. Complex evolutionary trajectories of sex chromosomes across bird taxa. Science. 2014;346:1246338.

30. Harris RS. Improved pairwise alignmen of genomic DNA. Ph.D. Thesis: The Pennsylvania State University; 2007.

31. Kiełbasa SM, Wan R, Sato K, Horton P, Frith MC. Adaptive seeds tame genomic sequence comparison. Genome Res. 2011;21:487-93.

32. Noé L, Kucherov G. YASS: enhancing the sensitivity of DNA similarity search. Nucleic Acids Res. 2005;33(SUPPL. 2):540-3.

33. Sætre G-P, Ravinet M. Evolutionary genetics. 1st edition: Oxford University Press; 2019.

34. Jobling M, Hollox E, Hurles M, Kivisild T, Tyler-Smith C. Human evolutionary genetics. 2nd edition: Garland Science, Taylor \& Francis Group, LLC; 2014.

35. Hooper DM, Price TD. Chromosomal inversion differences correlate with range overlap in passerine birds. Nat Ecol Evol. 2017;1:1526-34.

36. Xu L, Auer G, Peona V, Suh A, Deng Y, Feng S, et al. Dynamic evolutionary history and gene content of sex chromosomes across diverse songbirds. Nat Ecol Evol. 2019:3:834-44.

37. Fowler BLS, Buonaccorsi VP. Genomic characterization of sex-identification markers in Sebastes carnatus and Sebastes chrysomelas rockfishes. Mol Ecol. 2016:25:2165-75.

38. Gamble T, Zarkower D. Identification of sex-specific molecular markers using restriction site-associated DNA sequencing. Mol Ecol Resour. 2014;14:902-13.

39. Fridolfsson $A-K$, Ellegren $H$. A simple and universal method for molecular sexing of non-ratite birds. J Avian Biol. 1999;30:116-21.

40. Sim SC, van Deynze A, Stoffel K, Douches DS, Zarka D, Ganal MW, et al. High-density SNP genotyping of tomato (Solanum lycopersicum L.) reveals patterns of genetic variation due to breeding. PLoS One. 2012;7:1-18.

41. Li H, Ralph P. Local PCA shows how the effect of population. Genetics 2019;211:289-304.

42. Otto SP, Pannell JR, Peichel CL, Ashman TL, Charlesworth D, Chippindale AK, et al. About PAR: the distinct evolutionary dynamics of the pseudoautosomal region. Trends Genet. 2011;27:358-67.

43. Smeds L, Warmuth V, Bolivar P, Uebbing S, Burri R, Suh A, et al. Evolutionary analysis of the female-specific avian W chromosome. Nat Commun. 2015:6:7330.

44. Huylmans AK, Toups MA, MacOn A, Gammerdinger WJ, Vicoso B. Sexbiased gene expression and dosage compensation on the artemia franciscana Z-chromosome. Genome Biol Evol. 2019;11:1033-44.

45. Sigeman H, Ponnikas S, Hansson B. Whole-genome analysis across 10 songbird families within Sylvioidea reveals a novel autosome-sex chromosome fusion. Biol Lett. 2020;16:20200082.

46. Lewin HA, Robinson GE, Kress WJ, Baker WJ, Coddington J, Crandall KA et al. Earth BioGenome project: sequencing life for the future of life. Proc Natl Acad Sci. 2018:115:4325-33.

47. Helander B, Hailer F, Vilà C. Morphological and genetic sex identification of white-tailed eagle Haliaeetus albicilla nestlings. J Ornithol. 2007; 148:435-42.

48. Thermo Fisher. Thermo Scientific GeneJET Genomic DNA Purification Kit \#K0721, \#K0722. 2016; October:1-8. https://www.thermofisher.com/docum ent-connect/document-connect.html?url=https\%3A\%2F\%2Fassets.therm ofisher.com\%2FTFS-Assets\%2FLSG\%2Fmanuals\%2FMAN0012667_GeneJ ET_Whole_Blood_Genomic_DNA_Purification_Mini_Kit_UG.pdf\&title= VXNlciBHdWIkZTogR2VuZUpFVCBXaG9sZSBC. Accessed 14 Feb 2020. 
49. Illumina. TruSeq ${ }^{\circledR}$ Nano DNA Library Prep. 2015. https://support.illumina. com/content/dam/illumina-support/documents/documentation/chemi stry_documentation/samplepreps_truseq/truseqnanodna/truseq-nanodna-library-prep-guide-15041110-d.pdf. Accessed 14 Feb 2020.

50. Hillier LW, Miller W, Birney E, Warren W, Hardison RC, Ponting CP, et al. Sequence and comparative analysis of the chicken genome provide unique perspectives on vertebrate evolution. Nature. 2004;432:695-716.

51. Catchen J, Hohenlohe PA, Bassham S, Amores A, Cresko WA. Stacks: an analysis tool set for population genomics. Mol Ecol. 2013;22:3124-40.

52. Catchen JM, Amores A, Hohenlohe P, Cresko W, Postlethwait JH, De Koning D-J. Stacks: Building and genotyping loci de novo from short-read sequences. G3 Genes|Genomes|Genetics. 2011;1:171-82.

53. Babraham Bioinformatics. FastQC. 2010. http://www.bioinformatics.babra ham.ac.uk/projects/fastqc/. Accessed 16 Jun 2016.

54. Schubert M, Lindgreen S, Orlando L. AdapterRemoval v2: rapid adapter trimming, identification, and read merging. BMC Res Notes. 2016;9:88.

55. Li H, Durbin R. Fast and accurate short read alignment with burrowswheeler transform. Bioinformatics. 2009;25:1754-60.

56. Li H, Handsaker B, Wysoker A, Fennell T, Ruan J, Homer N, et al. The sequence alignment/map format and SAMtools. Bioinformatics. 2009;25:2078-9.

57. Quinlan AR, Hall IM. BEDTools: a flexible suite of utilities for comparing genomic features. Bioinformatics. 2010;26:841-2.

58. Eggertsson HP, Jonsson H, Kristmundsdottir S, Hjartarson E, Kehr B, Masson G, et al. Graphtyper enables population-scale genotyping using pangenome graphs. Nat Genet. 2017;49:1654-60.

59. Eggertsson HP, Kristmundsdottir S, Beyter D, Jonsson H, Skuladottir A, Hardarson MT, et al. GraphTyper2 enables population-scale genotyping of structural variation using pangenome graphs. Nat Commun. 2019;10:1-8.

60. Danecek P, Auton A, Abecasis G, Albers CA, Banks E, DePristo MA, et al. The variant call format and VCFtools. Bioinformatics. 2011;27:2156-8.

61. Li H. A statistical framework for SNP calling, mutation discovery, association mapping and population genetical parameter estimation from sequencing data. Bioinformatics. 2011;27:2987-93.

62. Meisner J, Albrechtsen A. Inferring population structure and admixture proportions in low-depth NGS data. Genetics. 2018;210:719-31.

63. Korneliussen TS, Albrechtsen A, Nielsen R. ANGSD: analysis of next generation sequencing data. BMC Bioinform. 2014;15:356.

64. da Fonseca RR, Albrechtsen A, Themudo GE, Ramos-Madrigal J, Sibbesen $J A$, Maretty L, et al. Next-generation biology: sequencing and data analysis approaches for non-model organisms. Mar Genomics. 2016;30:3-13.

65. R core team. R: a language and environment for statistical computing. 2020.

66. Chen H, Boutros PC. VennDiagram: a package for the generation of highly-customizable Venn and Euler diagrams in R. BMC Bioinformatics. 2011;12:35.

67. Hansen CCR, Baleka S, Guðjónsdóttir SM, Rasmussen JA, Ballesteros JAC, Hallgrimsson GT, et al. Distinctive mitogenomic lineages within populations of white-tailed eagles. Ornithology. 2021;139:1-14.

\section{Publisher's Note}

Springer Nature remains neutral with regard to jurisdictional claims in published maps and institutional affiliations.

Ready to submit your research? Choose BMC and benefit from:

- fast, convenient online submission

- thorough peer review by experienced researchers in your field

- rapid publication on acceptance

- support for research data, including large and complex data types

- gold Open Access which fosters wider collaboration and increased citations

- maximum visibility for your research: over $100 \mathrm{M}$ website views per year

At BMC, research is always in progress.

Learn more biomedcentral.com/submissions 